\title{
Lower Lip Reconstruction Using Unilateral Nasolabial Gate Flap (Fujimori Technique)
}

\author{
lacob Alina, Bögözi B \\ Department of Oral and Maxillofacial Surgery, Faculty of Dental Medicine, University of Medicine and Pharmacy, Tîrgu Mureș, România
}

\begin{abstract}
Background: In the medical literature there are multiple reconstructive procedures for small and medium size defects of the lower lip, but only a few methods for larger defects involving the whole lower lip. Choosing the repairing procedure for larger lower lip defects must take into account two aspects: flap or flaps used should be local flaps, and suture lines should correspond to the natural facial creases or follow the functional lines of different facial aesthetic units. Finally, the flap or flaps should be large enough to restore the entire postexcisional defect. However, the more tissue is lost from the lower lip, the more challenging the reconstruction is from a functional and cosmetic point of view. Material and methods: During the last 2 years in the Oral and Maxillofacial Surgery Department of the County Emergency Clinical Hospital in Tîrgu Mureș, the unilateral Fujimori technique has been successfully used for the reconstruction of large lower lip defects in 4 patients with extended, neglected squamous cell carcinomas involving almost the whole lower lip tissue. These patients underwent complex surgical and oncological treatment. Surgery was performed in two stages: first, excision of the tumor and immediate lip reconstruction, and then removal of submandibular lymph nodes (level I), in the second stage.

Results: The aesthetic and functional recovery of these patients was very good, with no need of other subsequent surgical corrections.

Conclusions: The postoperative reconstruction of large defects of the lower lip is far from optimal, but it is very important to restore an adequate muscle function, the lip continence and a satisfactory facial appearance.
\end{abstract}

Keywords: lip reconstruction, naso-labial gate flap, large lip tissue loss, squamous cell carcinoma

Received: 1 January 2013

\section{Introduction}

Squamous cell carcinoma is the most common malignant tumor of the lower lip. Invasive squamous cell carcinoma seldom arises de novo, in most of the cases (over 60\%) invasive tumoral forms are preceded by one of the premalignant lesions (actinic cheilitis or leukoplakia), which are considered by some authors as carcinoma in situ forms. Being an epithelial cancer itself, hypothetical all invasive forms of squamous cell carcinoma should have followed by an in situ phase [1].

The treatment of squamous cell carcinomas of the lower lip is primarily surgical and consists of tumor excision of the lower lip, followed by immediate reconstruction (in the same operative session). Small defects (up to $1 / 3$ of the entire lower lip) represent no problem for surgical reconstruction, the defect being closed by direct suture using various technical methods with excellent functional and cosmetic results [2].

For larger lower lip defects, reconstruction becomes difficult because it is important to restore the anatomy of the lip (the anatomical structures of the lip are unique - vermilion, $\mathrm{m}$. orbicularis oris) and its proper function. It is important to use the remaining labial tissue for reconstruction, associated with methods that bring innervated muscle to restore the oral sphincter, and thus, labial continence. Sensitive function of the lip is difficult to be preserved in these cases.

Correspondence to: Bálint Bögözi

E-mail: bogozibalint@yahoo.com
Many surgical techniques have been described to repair larger defects of the lower lip, each with its advantages and disadvantages: Karapandzic flap, Bernard-Burow's procedure, Gillies fan flap, advanced/rotated regional flaps, free flap reconstruction $[3,4]$.

\section{Material and methods}

The number of patients with extensive tumors of the lower lip is relatively small due to early diagnosis and the surgical treatment which is thus performed as soon as possible. However, some patients tend to neglect their condition and in such cases the treatment must be done in advanced stages (T3-T4), when the lower lip is heavily infiltrated by the tumor (over $2 / 3$ ).

On the other hand, being a malignant tumor, the lower lip carcinoma tends to invade submandibular and submental lymph nodes in all stages, but especially in those advanced. In these cases, pre- and postoperative metastatic lymph node invasion can be effectively monitored using ultrasound or CT examination, or performing fine needle aspiration for cytologic evaluation. In most situations, cytology can accurately differentiate, in a few hours, if there is an inflammatory or a metastatic lymph node [5].

In our clinic many reconstructive techniques are used to repair extensive defects of the lower lip, but in the last two years we have successfully used the Fujimori technique to reconstruct major defects of the lower lip.

The Fujimori technique consists of performing two nasolabial compound island flaps, skin-muscle-mucosa, of 


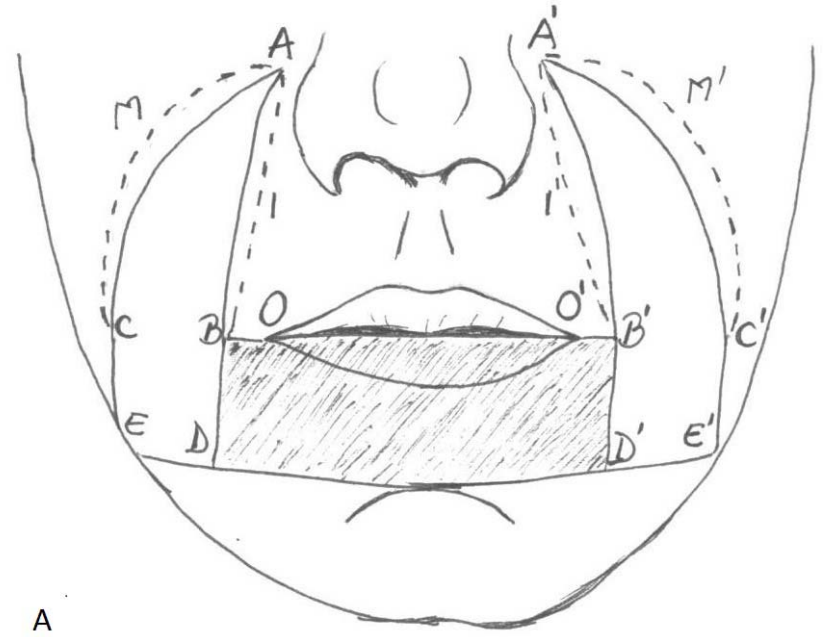

Fig. 1. The operative technique (author's drawing)

triangular shape, about $3 \mathrm{~cm}$ wide on the cutaneous part. Mucosal incisions will form a flap wider (about $4 \mathrm{~cm}$ ) on the inside - the mucosal excess is necessary for vermilion reconstruction. At the base of the flap the incisions are made only in the skin and subcutaneous tissue, muscle and mucosa remaining intact. Thus, the prepared flap contains innervated muscle (orbicularis oris, canine, zygomatic minor and major, rizorius, triangular and buccinator) and angular artery.

The flap is then mobilized and rotated into the defect site. The suture lines are placed in the nasolabial fold, labio-mentonier groove, the vermilion edge, and intraorally along the vestibular sulcus, respectively. Suture is made in 4 layers: mucosal, muscular, subcutaneous and skin. Mucosal excess from the upper edge of the flap is used to restore the vermilion. Mobilizing the innervated check muscles

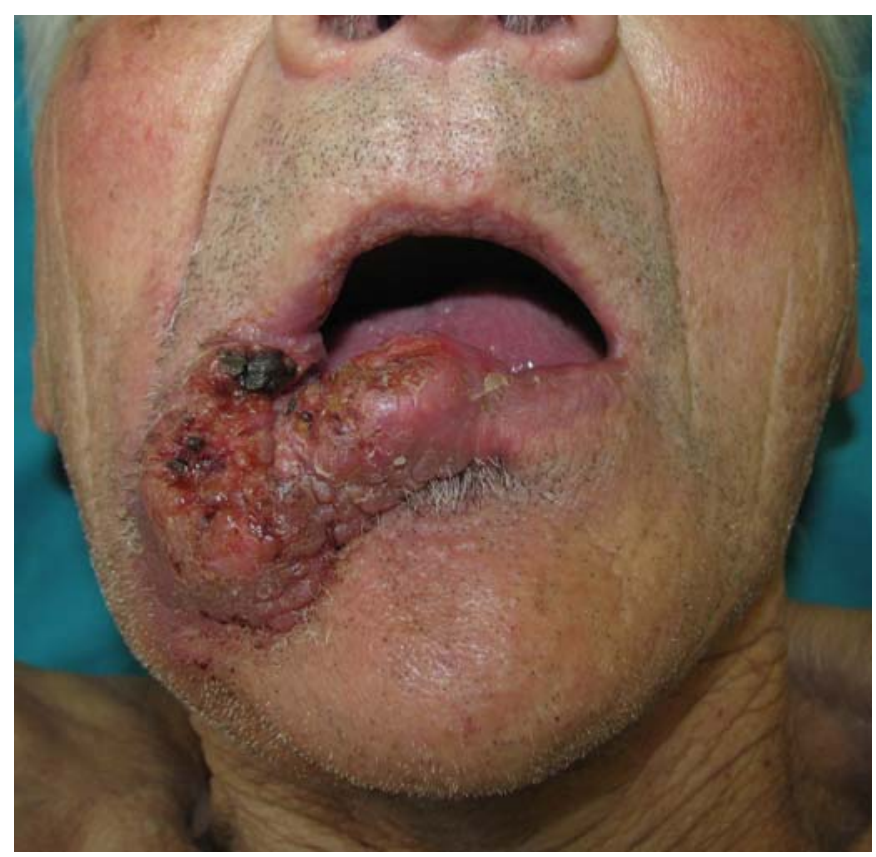

Fig. 2. Preoperative aspect

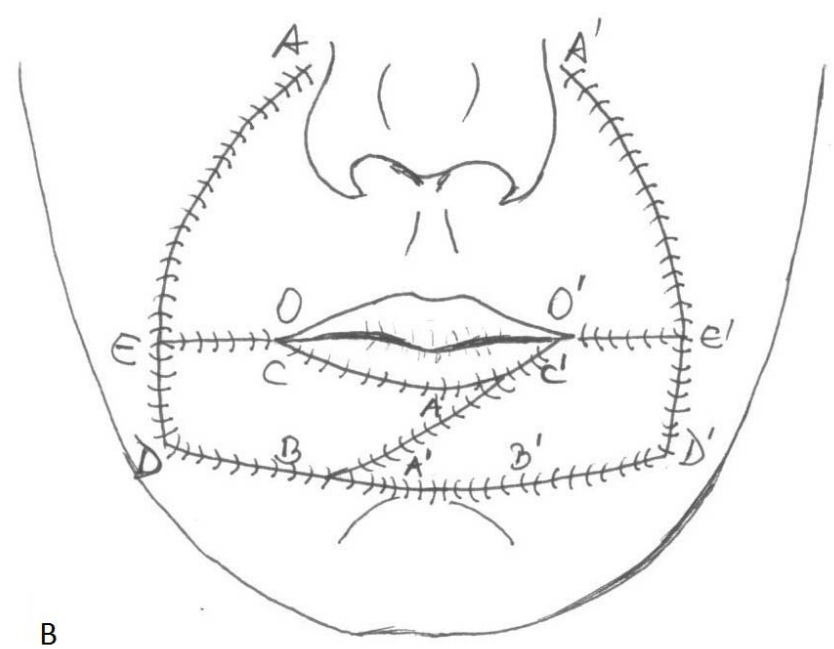

and the mucosal lining over the lower lip contributes to partial recovery of sensitivity and motor function (which is considered debatable by some authors) [6].

The operative technique is presented in Figure 1, and described as it follows, according to Fujimori: distance $O B$ $=\mathrm{O}^{\prime} \mathrm{B}^{\prime}=0.5$ to $1.0 \mathrm{~cm}$ in normal patients, but it can be extended to $2 \mathrm{~cm}$ in the case of older patients with wrinkled face. Distance $B C=B^{\prime} C^{\prime}=3 \mathrm{~cm}$. Skin incision along $A B$ and $A C$, and $A^{\prime} B^{\prime}$ and $A^{\prime} C^{\prime}$ respectively, should be made through-and-through into the mouth. Skin incision along CE and ED, and C'E' and E'D' respectively, should be made only through subcutaneous tissue. Incision in the mucosa membrane is performed along the dotted lines (AIB, AmC, A'I'B', A'm'C') that run laterally to the skin incision lines $\left(A B, A C, A^{\prime} B^{\prime}, A^{\prime} C^{\prime}\right)$. The dotted area is undermined subcutaneously (Figure 1A). Skin suture lines run along the nasolabial fold and mentolabial groove centrally (Figure 1B) [6].

In the cases operated in our clinic, due to the presence of a small remaining portion of the lower lip, it was not necessary to perform bilateral Fujimori gate flaps, the reconstructive method being used only unilaterally.

\section{Results}

Fujimori unilateral gate flap was successfully used in our department on 4 patients with major defects of the lower lip. About $70-75 \%$ of lip was lost after excision of the extensive tumors. All 4 patients were male, aged between 62 and 74 years.

In all cases, the flaps survived without complications. The flap rotation and positioning into defect was performed each time without "dog-ears" and the vascularization supplied by the angular artery and satellite veins was excellent. The initial postoperative edema withdrew gradually; the healing period was relatively short (about 3-4 weeks). The color and texture of the nasolabial tissue used in this reconstructive procedure is almost identical to the 


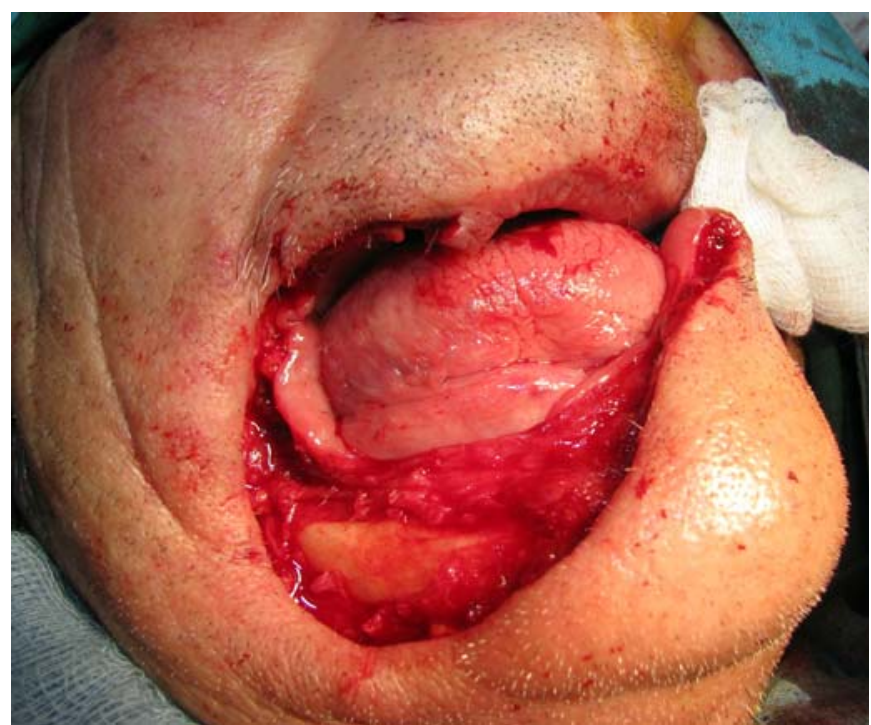

Fig. 3. The postexcisional defect

normal lower lip. No further corrections were required, the patients being satisfied with their postoperative facial appearance. Scars in the donor area were perfectly integrated and morbidity at this level was nil.

The oral sphincter was competent and the functional aspect was considered excellent, without loss of saliva or food. Mouth opening was appreciated to be good in all cases, allowing proper feeding and oral hygiene. Sensitivity and motor function were well preserved.

To illustrate these results, we present one of our clinical cases: 68 years old male patient, who referred to our clinic with an extensive lower lip tumor, affecting $2 / 3$ of the lower lip, the right oral commissure and mental region (Figure 2). The postexcisional defect after surgical removal of the tumor can be seen in Figure 3. For the reconstruction of the lower lip an unilateral Fujimori gate flap was

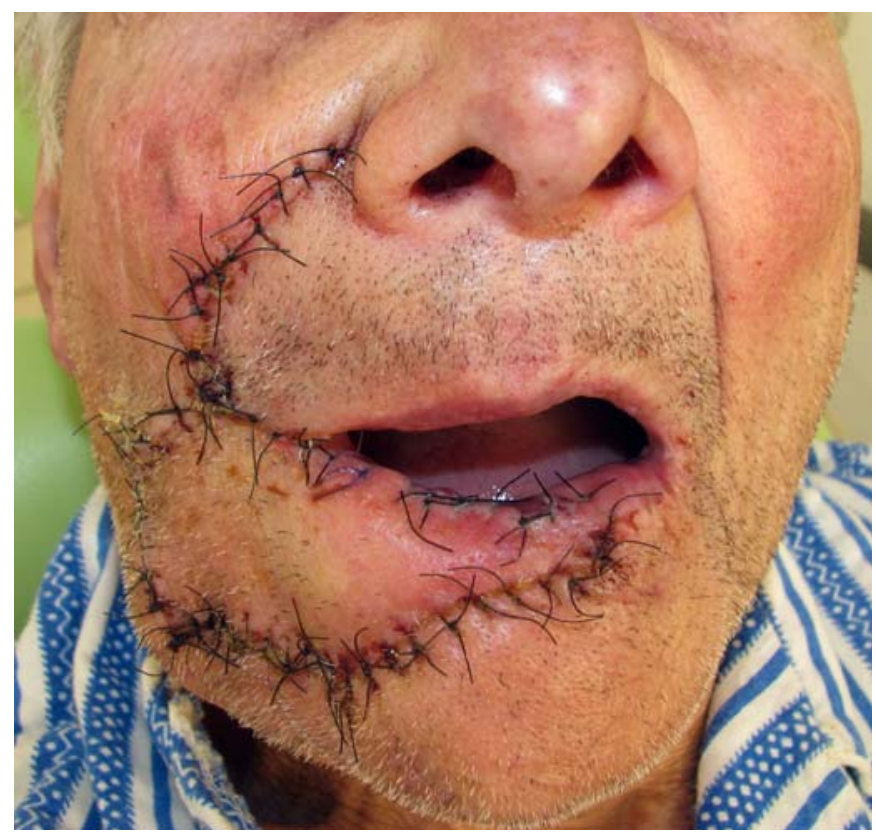

Fig. 5. The postoperative aspect, two days after

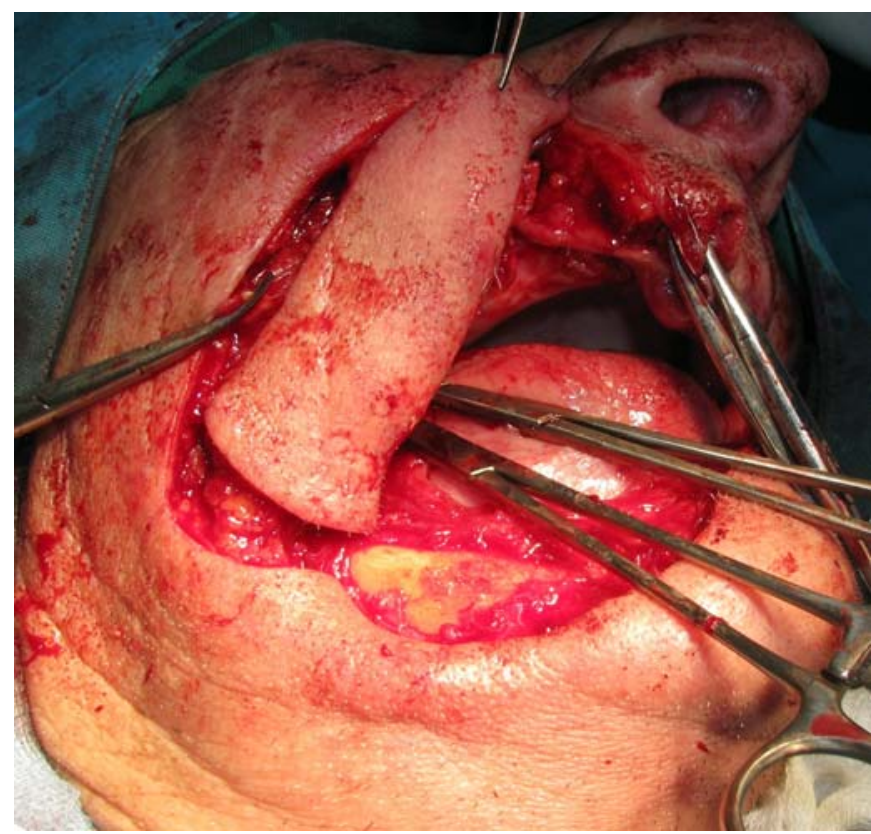

Fig. 4. The mobilized flap

used (Figure 4). The postoperative aspect after 2 days and 1 month are presented in Figures 5 and 6.

Submandibular lymph nodes excision was performed after 3-4 weeks (the second operative stage). Subsequently, oncological treatment (radiotherapy) was indicated to all patients. One patient had recurrent squamous cell carcinoma of the lower lip, and was already operated once on the lower lip and submandibular lymph nodes were removed 5 years before. On this patient, only tumor recurrence was excised followed by lower lip reconstruction.

Another patient refused removal of submandibular lymph nodes, so he underwent oncological treatment only. The other 2 patients received standard treatment.

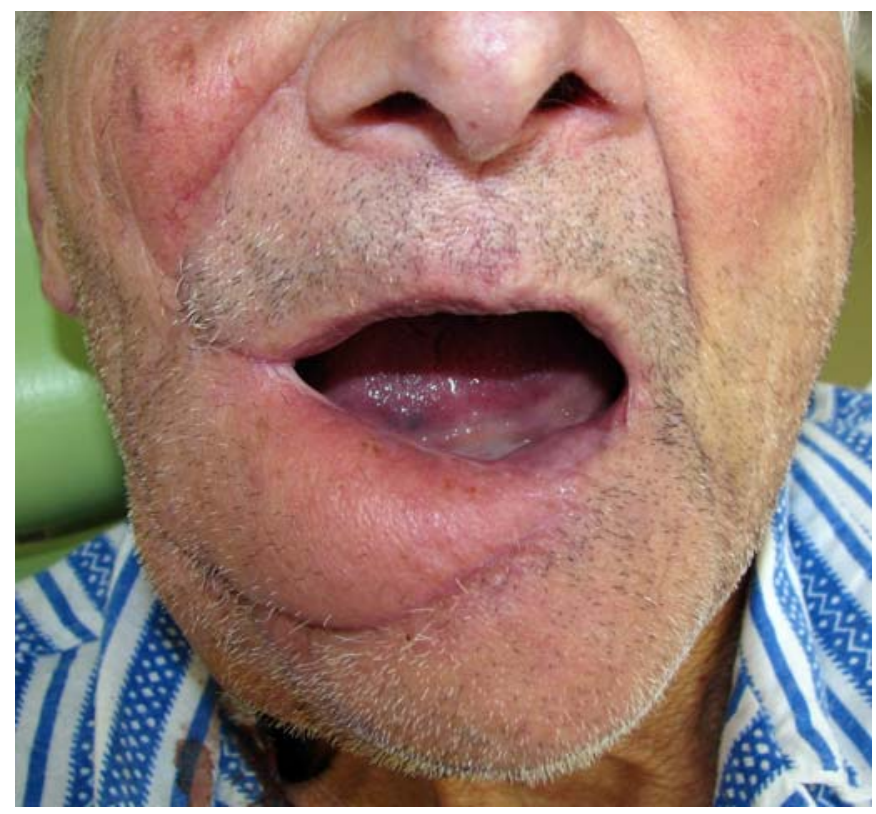

Fig. 6. The postoperative aspect one month later 


\section{Discussions}

Although the incidence of invasive squamous cell carcinoma of the lower lip is relatively high (about $90 \%$ of the lip tumors and $30 \%$ of the whole oral cavity tumors), rarely the lesion exceeds $1-2 \mathrm{~cm}$ in the largest diameter (T1). Usually, patients are referred in early stages for surgical treatment.

After the tumor removal of the lower lip, reconstruction should be done with the same kind of tissue. This target can be reached in lower lip defect up to $1 / 3$ of the whole lip. Extensive defects cannot be ideally repaired due to the lack of similar tissues. In these cases, the chosen method for reconstruction should aim: maintaining of proper function and sensitivity of oral sphincter, in order to prevent the loss of food and saliva, allowing sufficient mouth opening and having an acceptable appearance [2].

The surgeon has only several options in order to perform lower lip reconstruction in large postoperative defects: the Bernard-Burow's technique, Gillies fan-flap, Karapandzic technique or nasolabial flaps (Fujimori or von Bruns procedure). Only in severe cases with entire lower lip and surrounding tissue loss, free vascularised flap transfer should be considered.

Some authors indicate Karapandzic flap, unilateral or bilateral, as a first option to repair extensive lower lip defects (up to $80 \%$ of the lip). This procedure uses innervated musculo-cutaneous arterial flap that contain the remaining labial and cheek tissue, which are then advanced and rotated to the midline in order to reconstruct the lower lip and to keep its sensitivity. Postoperative healing, in most cases result in microstomia and commissural distortion that require further corrections due to significant functional limitations $[2,3,7]$.

Gillies fan flap is another reconstructive option that can be performed unilateral or bilateral. This procedure rotates cheek flaps for the reconstruction of the lower lip, but their structure contains denervated muscles, so there is no sensitivity in the newly formed lip. On the other hand, secondary procedures are also necessary in order to correct the postoperative microstomia $[4,8]$.

Bernard-Burow's procedure reconstructs the lower lip by advancing the adjacent cheek tissue, but large amounts of skin and subcutaneous tissue are sacrificed at the labiomental and nasolabial folds with motor and sensitivity loss. This results in a tight lower lip and significant perioral deformity. This procedure can be used in association with common nasolabial flap (von Bruns) [3,4].

Removal of extremely large tumors requires distance and free flaps for facial reconstruction, but they cannot recreate a functional oral sphincter and the harmony of the face. Patients may have some donor site morbidity and the operation time is quite long [3].
Nasolabial gate flap described by Fujimori is an improvement over the procedures described above. When properly performed, the flap is rotated into the labial defect without "dog-ear" forming. Blood supply of the flap is excellent due to angular artery, and drainage is done through its satellite veins (Figure 5). Reconstruction procedure is carried out in one surgical stage. No further correction is necessary. The functional and esthetic benefits seem to be superior to other methods [3].

The surgical technique is relatively simple, accessible and safe, avoiding the complex problems related to free flap transfer. Nasolabial skin relocated to the lower lip is in harmony with the surrounding tissue, giving a pleasant facial aspect (Figure 6).

\section{Conclusions}

In our opinion, Fujimori technique performed either unilateral or bilateral could be considered an effective option in reconstruction of large defects of the lower lip (over $50 \%$ of the lip), since this technique allows proper lip sensitivity and muscle function recovery, oral continence and communication abilities, better than other reconstructive methods.

Reconstruction of large defects of the lower lip is far from ideal, but it is very important to restore a proper lip function that involves recovering oral competence, lip sensitivity which is mandatory to an adequate nutrition. Also, one should not neglect the role of the lower lip in speaking and expressing emotions, which allows social integration, together with a pleasant facial appearance of each individual.

\section{References}

1. Anadolu-Brasie R, Patel A, et al. Squamous Cell Carcinoma of the Skin, in Nouri K: Skin Cancer. McGraw Hill Medical, New York, Chicago, San Francisco, Lisbon, London, Madrid, Mexico City, Milan, New Delhi, San Juan, Seoul, Singapore, Sidney, Toronto, 2008;86-114.

2. Mohamed AR. Lower Lip Reconstruction after Tumor Resection; a Single Author's Experience with Various Methods. Journal of the Egyptian Nat. Cancer Inst. 2006;18(4):323-333.

3. Samy Ahmad Shehab El-Din. Lower Lip Reconstruction with Fujimori Gate Flaps. Egypt. J Plast Reconstr Surg. 2003;27(2):319-324.

4. Sajjadian A. Lip Reconstruction Procedures Treatment \& Management. Available online at emedicine.medscape.com/article/1288447-treatment. Updated Nov. 20, 2011.

5. Johnson JT. Fine-Needle Aspiration of Neck Masses. Available online at emedicine.medscape.com/article/1819862-overview. Updated April 2012.

6. Fujimori R. Nasolabial (Gate) Skin-Muscle-Mucosal Flap to Lower Lip, in Strauch B, Vasconez LO, Hall-Findaly EJ (eds): Grabb's Encyclopedia of Flaps, 2nd ed. Lippincott-Raven, Philadelphia, 1998;586.

7. Karapandzic M. Innervated Musculocutaneous Lip and Cheek Flaps, in Strauch B, Vasconez LO, Hall-Findaly EJ (eds): Grabb's Encyclopedia of Flaps, 2nd ed. Lippincott-Raven, Philadelphia, 1998;615.

8. Mullin WR, Millard Jr. DR. Fan Flaps, in Strauch B, Vasconez LO, HallFindaly EJ (eds): Grabb's Encyclopedia of Flaps, 2nd ed. LippincottRaven, Philadelphia, 1998;61. 